\title{
Co-compostage de boues de vidange domestiques avec des déchets maraîchers et des déchets de poissons à Dakar (Sénégal)
}

\author{
Maïmouna $\mathrm{LO}^{1}$, El hadji Mamadou SONKO ${ }^{1 *}$, Diomaye DIENG ${ }^{1}$, Saliou NDIAYE ${ }^{2}$, \\ Cheikh DIOP ${ }^{1}$, Alsane SECK $^{1}$ et Amadou GUEYE ${ }^{1}$ \\ ${ }^{1}$ Laboratoire d'Etude Environnemental des Milieux Urbains (LEEMUR); Institut des Sciences de \\ l'Environnement, Faculté des Sciences et Technique, Université Cheikh Anta Diop de Dakar. BP 5005, \\ Dakar-Fann, Sénégal. \\ ${ }^{2}$ Ecole Nationale Supérieure d'Agriculture, ENSA Km 7 route de Khombole, BP A 296 Thiès RP \\ *Auteur correspondant ; E-mail: elmsonko@gmail.com / elhadjimamadou.sonko@ucad.edu.sn; \\ Tel:+221 775717518 .
}

\section{RESUME}

Les boues de vidange ont des valeurs fertilisantes importantes mais elles renferment beaucoup d'agents pathogènes qui limitent leur utilisation en agriculture. C'est pour contribuer à l'amélioration de la valeur agronomique et la réduction des risques sanitaires que la présente étude s'est proposée de mettre en place une technique de valorisation à moindre coût et accessible. Cette étude s'est appuyée sur le co-compostage des boues de vidange avec des co-substats d'origine animale et végétale. Une série d'analyse des paramètres physico-chimiques, microbiologique et parasitaire a permis de suivre la maturité du compost, d'évaluer la valeur agronomique et d'apprécier les teneurs en coliformes fécaux et en œufs d'helminthes. Au terme des expérimentations et des analyses, l'humidité est respectée pour les traitements avec les co-substrats d'origine végétale mais des variations ont été notées avec les traitements qui ont reçu les co-substrats d'origine animale. Les $\mathrm{pH}$ se situent entre 6 et 7 et les conductivités électriques sont inférieures à $2000 \mathrm{~s} / \mathrm{cm}$. Les rapports $\mathrm{C} / \mathrm{N}$ sont inférieurs à 12. Les éléments nutritifs sont intéressants avec l'azote $(1,3$ et $2,2 \%)$, le phosphore $(0,2$ et $0,5 \%)$, et le potassium $(0,07$ à $0,12 \%)$. Les concentrations en coliformes fécaux sont de 20000 à 37000 $\mathrm{UCF} / 100 \mathrm{~g}$, et les œufs d'Ascaris vivants sont autour de 1,2 à 3,4 nombre d'œufs/g. Les résultats de cette étude ont, en effet, montré que tous les composts étaient matures dans tous les andains. Toutefois, ces composts ne sont sûrs du point de vue sanitaire avec des concentrations en coliformes fécaux supérieures aux normes internationales.

(C) 2019 International Formulae Group. All rights reserved.

Mots clés: Boues de vidange, valeur agronomique, co-compostage, maturité.

\section{Co-composting of faecal sludge with vegetable and fish waste in Dakar (Senegal)}

\begin{abstract}
Faecal sludge has important fertilizing values but it contains many pathogens agents that limit its use in agriculture. To contribute to the improvement of their agronomic value and the reduction of health risks, this study proposed to implement a low-cost valuing technique accessible through the co-composting of sewage sludge with co-substats of animal and plant origin. A series of analyses of physico-chemical, microbiological
\end{abstract}


and parasitic parameters made it possible to monitor the maturity of the compost, assess its agronomic value and assess the levels of faecal coliforms and roundworm eggs. At the end of the experiments, humidity is respected for treatments with co-substrates of plant origin but variations have been noted with treatments that have received co-substrates of animal origin. The $\mathrm{pH}$ values are between 6 and 7 and the electrical conductivity is below $2000 \mathrm{~s} / \mathrm{cm}$. C/N ratios below 12. Composts are rich in nutrients such as nitrogen (1.3 to 2.2\%), phosphorus ( 0.2 to $0.5 \%)$, and potassium $(0.07$ to $0.12 \%)$. Fecal coliform concentrations are 20,000 to 37,000 $\mathrm{UCF} / 100 \mathrm{~g}$, and living Ascaris eggs are around 1.2 to 3.4 number of eggs/g. The results of this study showed that all composts were mature in all windrows but are not safe from a health point of view with concentrations of fecal coliforms and helminth eggs exceeding international standards.

(C) 2019 International Formulae Group. All rights reserved.

Keywords: Faecal sludge, co-composting, maturity, health quality, agronomic value.

\section{INTRODUCTION}

A l'image des autres pays en voie de développement, le Sénégal fait face à un grand problème d'assainissement, de par sa forte croissance démographique ANDS (2013). Ainsi une grande production de boues de vidange a lieu dans une grande partie du territoire. Cette situation pousse la population à adopter des méthodes peu conventionnelles pour gérer les déchets issus des latrines et autres fosses septiques (Gning et al., 2017). Face à ce fléau, l'Etat a pris des engagements en investissant dans plusieurs secteurs comme la couverture des besoins en assainissement depuis 2005. Ainsi, les taux d'accès à l'assainissement ont graduellement augmenté, des stations d'épuration ou de traitement des boues de vidange ont été construites ou sont en construction dans les régions à forte densité de population.

Parallèlement, la pauvreté des sols pousse les populations à utiliser directement les produits issus de la vidange dans le maraichage (Kone, 2016). Selon Keffala et al, (2012), la réutilisation des eaux usées et des boues sans traitement devient de plus en plus importante. Cette pratique est connue à Dakar dans la zone des Niayes qui représente $80 \%$ du maraichage au Sénégal. Elle se renforce de plus en plus du simple fait que, selon certains maraichers, elle permet une économie d'eau et d'intrants (Gaye et Niang, 2010).

La réutilisation des eaux usées et des boues est motivée par le fait que ces produits sont riches en matières minérales (Seck, 2016) et organiques qui peuvent être bénéfiques pour le développement des plantes et de la structure du sol (Gaye et Niang, 2010 ; Niang et al., 2012). Toutefois, cette pratique peut constituer un risque sanitaire pour la population. En effet les eaux usées présentent une forte concentration en œufs d'helminthes (Ndiaye, 2009). Ce risque est plus prononcé dans le cas où l'utilisation se fait de façon directe car les concentrations en germes pathogènes sont très élevées dans les eaux usées (Maya et al., 2012). Pour tirer un meilleur profit des potentialités agronomiques des boues de vidanges et en même temps protéger les populations des risques liés à leur réutilisation, leur traitement hygiénique semble donc inévitable. Cependant les technologies de traitement applicables aux boues d'épurations peuvent être utilisées pour traiter les boues fécales (Kouawa, 2017). Le défi est alors d'élaborer des options de traitement à faible coût dans le but de réutiliser les sous-produits. Parmi les technologies disponibles pour recycler les déchets solides organiques, le compostage est souvent présenté comme une technique accessible et à faible coût pour convertir les déchets en un produit utilisable pour amender le sol (Ruggieri et al., 2008b). Les co-subtrats compostés avec les boues de vidange peuvent toutefois se comporter différemment selon leur nature. En effet plusieurs études ont montré que les vitesses de dégradation des matières grasses, des sucres et des protéines étaient différentes et les produits finaux souvent différents (Ruggieri et al., 2008b). Ainsi notre étude se propose de tester deux types de co-substrats; des déchets d'origine végétale (déchets maraichers) et des déchets d'origine animale (déchets de poissons). 


\section{MATERIEL ET METHODES \\ Dispositif expérimental}

Un tas de compost a été créé selon la méthode de l'andain au niveau de la Station de traitement des boues de vidange de Cambérène (Dakar, Sénégal). Sept (7) traitements (tas) ont été constitués comme suit :

$>\mathrm{T} 0$ : Témoin, constitué uniquement de boues de vidange (BV);

> T1a: mélange de 2 volumes $\mathrm{BV}+$ 1 volume de déchets maraîchers (DM)

> T2a : mélange de 1 volume $\mathrm{BV}+1$ volume DM

$>$ T3a: mélange de 1 volume $\mathrm{BV}+$ 2 volumes DM

$>$ T1b: mélange de 2 volumes $\mathrm{BV}+$ 1 volume de déchets poissons (DP)

$>\mathrm{T} 2 \mathrm{~b}$ : mélange de 1 volume $\mathrm{BV}+1$ volume DP

$>\mathrm{T} 3 \mathrm{~b}$ : mélange de 1 volume $\mathrm{BV}+2$ volumes DP

Les différents matériaux ont été mélangés pour former des tas coniques d'environ $1 \mathrm{~m}$ de diamètre de base sur une hauteur d'environ $50 \mathrm{~cm}$. Pour éviter la lixiviation et les pertes de nutriments, les andains ont été réalisés sur une surface étanche et un imperméable a été posé audessus.

La durée totale du processus de compostage était de trois mois. Les andains étaient retournés tous les dix jours comme l'ont suggéré Cofie et al. (2009), pour favoriser une dégradation plus ou moins homogène de la matière organique. Le retournement permet aussi d'aérer les andains. A chaque retournement, les andains sont arrosés afin de maintenir l'humidité dans des proportions compatibles avec l'activité biologique de l'ordre de $40 \%$ à $60 \%$ selon Fourti (2013). Durant l'expérimentation les températures ambiantes ont varié entre 18 et $23{ }^{\circ} \mathrm{C}$.

\section{Description des matériaux utilisés}

Les boues de vidange utilisées sont issues des lits de séchage plantés de la Station de Traitement des Boues de Vidange (STBV) de Cambérène. Elles proviennent des systèmes d'assainissement autonomes comme les fosses septiques. Elles sont amenées à la STBV par des camions de vidange. A ce niveau elles sont dépotées dans des décanteurs épaississeurs. Après une semaine de décantation, les boues sont pompées sur des lits de séchage non plantés où elles sont mises à sécher pendant deux semaines avant d'être collectées pour être compostées.

Les déchets de poisson utilisés ont été collectés au niveau du marché central aux poissons de Dakar (Sénégal). Ils sont essentiellement composés de têtes, de branchies, d'arrêtes, d'écailles et de viscères de poissons.

Les déchets maraichers ont été collectés au niveau du marché Gueule Tapée des Parcelles Assainies (Dakar/Sénégal). Il s'agit essentiellement de restes de légumes, d'épluchures de légumes, de tiges de manioc, de feuilles d'oseille, de feuilles de salades, de feuilles de choux et de gousses d'oignons en décomposition.

Dans cette étude, nous avons travaillé à la station d'épuration de Cambérène située à Dakar la capitale du Sénégal.

\section{Méthodes \\ Traitement des boues utilisées}

Les boues de vidange sont laissées en séchage sur les lits pendant trois semaines. A la fin de la période de séchage, les boues sont récoltées pour être co-compostées seules ou avec les déchets maraichers et les déchets de poissons. Les boues récoltées ont une siccité variant entre $40-70 \%$.

\section{Suivi de la qualité du compost Echantillonnage}

L'humidité et la matière organique ont été suivies durant tout le processus de compostage sur des échantillons collectés tous les dix jours, avant chaque retournement. Par contre, pour les autres paramètres, les prélèvements ont été réalisés après deux mois de compostage et à la fin du processus de compostage qui a duré 120 jours. Les échantillons analysés ont été prélevés au niveau de chaque andain à trois (3) endroits différents : la périphérie (au niveau des 
flancs), au centre de l'andain (au niveau superficiel, $0-10 \mathrm{~cm}$ de profondeur) au centre (au-delà de $10 \mathrm{~cm}$ de profondeur) (Cofie et al., 2009). Au niveau de chaque andain, les trois sub-échantillons prélevés ont été mélangés dans les mêmes proportions pour constituer l'échantillon à analyser.

Suivi du processus de compostage et de la qualité du compost

Détermination des paramètres physicochimiques

La température a été mesurée avec un thermomètre à compost de marque $\mathrm{BMG}$ ayant une gamme variant de 0 à $80{ }^{\circ} \mathrm{C}$. Les mesures ont été prises tous les jours, à partir du lendemain de la mise en place des andains.

L'humidité a été déterminée à travers la teneur en matière sèche par séchage à l'étuve à $105{ }^{\circ} \mathrm{C}$ jusqu'à un poids constant. Sur le même échantillon, la teneur en matière organique a été déterminée via la perte au feu par combustion au four à $550{ }^{\circ} \mathrm{C}$ pendant 3 heures (APHA, 2005).

Le $\mathrm{pH}$ et la conductivité ont été mesurés directement avec un pH-mètre GLP 21 Crison muni d'une électrode de verre dans des solutions obtenues en mettant en suspension le compost dans de l'eau distillée. Pour le $\mathrm{pH}$ de $20 \mathrm{~g}$ de compost ont été dilués dans $50 \mathrm{~mL}$ d'eau distillée et pour la conductivité, les $20 \mathrm{~g}$ de compost ont été dilués dans $200 \mathrm{~mL}$ d'eau distillée. Les ions $\mathrm{Ca}, \mathrm{Mg}$ ont été analysées par complexiométrie alors que le $\mathrm{Na}$, le $\mathrm{K}$ ont été analysé par photométrie. Le rapport $\mathrm{C} / \mathrm{N}$ a été calculé à partir des résultats d'analyses distincts du carbone et de l'azote. Le carbone a été analysé à partir de la méthode de Walkley et Black modifiée et l'azote a été analysé selon la méthode Kjeldahl. Le Phosphore a été déterminé par la méthode de dosage colorimétrique (Milin, 2012). Enfin, les acides humiques $(\mathrm{AH})$ et fulviques (AF) ont été analysés après fractionnement selon la méthode de l'International Humic Substances Society (IHSS).
Détermination des paramètres microbiologiques et parasitologiques

L'analyse des coliformes fécaux s'est faite par la méthode du dénombrement sur gélose (AFNOR). Les œufs d'Ascaris ont été déterminés selon la méthode développée par Water Research Commission (Palm et al., 2001).

\section{RESULTATS}

\section{Suivi du processus de compostage Evolution de l'humidité}

Les analyses de l'humidité au cours du processus de compostage ont montré que dans tous les andains de co-compostage de boues brutes et de déchets maraichers et, dans l'andain témoin l'humidité se situe entre 40 et $60 \%$ (Figure 1a). Par contre une forte variation de l'humidité est observée avec les traitements des boues de vidange avec les déchets de poissons (Figure 1b) se situant entre $15 \%$ et $60 \%$. En effet, dans les échantillons prélevés au niveau des andains ayant comme co-substrat les déchets de poissons, il y'a beaucoup de déchets solides persistants comme les os et les écailles de poissons. Ainsi la variation de l'humidité est beaucoup plus accentuée dans les traitements avec les déchets de poissons avec la nature des matériaux utilisés. En revanche les déchets maraichers ont des propriétés beaucoup plus absorbantes.

\section{Evolution de la température}

Dans les andains ayant comme cosubstrat les déchets maraichers la phase thermophile est observée durant les premiers jours de compostage avec une température maximale se situant entre 40 et $60{ }^{\circ} \mathrm{C}$ en fonction de la dose (Figure 2a). Cette phase thermophile a duré 8 jours.

Par contre dans les andains ayant comme co-substrat les déchets de poisson, la phase thermophile se met en place beaucoup plus lentement (Figure 2b). Les températures maximales sont plus élevées pour $\mathrm{T} 1 \mathrm{~b}, \mathrm{~T} 2 \mathrm{~b}$ et T3b lors de la phase thermophile montrant l'importance de la teneur en déchets de poisson. En effet, les températures maximales 
de la phase thermophile apparaissent entre les 10ème et 20ème jours de compostage au niveau des traitements $\mathrm{T} 1 \mathrm{~b}, \mathrm{~T} 2 \mathrm{~b}$ et $\mathrm{T} 3 \mathrm{~b}$. Les températures maximales apparaissent plus lentement dans les andains ou il y'a beaucoup plus de déchets de poissons. Dans les andains $\mathrm{T} 2 \mathrm{~b}$ et $\mathrm{T} 3 \mathrm{~b}$ les phases thermophiles sont beaucoup plus longues de l'ordre de 40 jours (entre les $20^{\text {ème }}$ et $60^{\text {ème }}$ jours de compostage) contre 10 jours (entre les $10^{\text {ème }}$ et $20^{\text {ème }}$ jours) et 5 jours (entre les $5^{\text {ème }}$ et $10^{\text {ème }}$ jours) pour respectivement $\mathrm{T} 1 \mathrm{~b}$ et $\mathrm{T} 0$. Les températures maximales enregistrées sont de l'ordre de $60{ }^{\circ} \mathrm{C}, 61{ }^{\circ} \mathrm{C}$ et $58{ }^{\circ} \mathrm{C}$ respectivement pour $\mathrm{T} 1 \mathrm{~b}, \mathrm{~T} 2 \mathrm{~b}$ et T3b.

\section{Variations du pH}

L'analyse de ce paramètre a montré des valeurs légèrement acides à neutre de 6 à 7 pour l'ensemble des andains (Figure 3 ). Elle montre que le $\mathrm{pH}$ n'a pas beaucoup varié au cours du compostage.

\section{Variation de la conductivité}

Les niveaux de conductivité électrique mesurés dans cette étude sont en baisse au cours du processus de compostage (Figure 4). En effet les traitements de boues de vidange et de déchets maraichers (T1a, T2a et T3a), ont enregistré des baisses de 2000-2500 $\mu \mathrm{s} / \mathrm{cm}$ à $1500-2000 \mu \mathrm{s} / \mathrm{cm}$ à la fin du compostage. Dans les andains ayant comme co-substrat les déchets de poissons (T1b, T2b et T3b), la conductivité a baissé de 2300-2800 $\mu \mathrm{s} / \mathrm{cm}$ à $1700-1900 \mu \mathrm{s} / \mathrm{cm}$. Aussi les boues brutes non compostées ont présentées des valeurs en conductivité électrique sont inférieures aux autres valeurs.

\section{Evolution de la teneur en matière organique}

La teneur en matière organique au cours du compostage dans les andains cocompostés avec les déchets maraîchers (Figure 5a) est passée de $70 \%$ de matières sèches à moins de $30 \%$ et ceux co-compostés avec les déchets de poissons (Figure $5 b$ ) observent une baisse beaucoup moins persistante allant d'environ $80 \%$ à moins de $40 \%$. Selon la nature des co-substrats utilisés, la dégradation des taux de matières organiques dans les traitements avec les déchets maraichers est beaucoup plus importante que dans les traitements avec les déchets de poissons

\section{Qualité agronomique des composts}

Les caractéristiques moyennes des composts obtenus à l'issue de cette étude sont consignées dans le Tableau 1. Tous les composts ont des $\mathrm{pH}$ légèrement acides (entre 6,2 et 6,6). Ces composts ont des rapports $\mathrm{C} / \mathrm{N}$ inférieurs à 12. Les rapports obtenus dans cette étude sont comparables et varient entre 8,4 et 10,6 quelle que soit la nature et la dose du co-substrat mélangé aux boues de vidange. La stabilisation a aussi été matérialisée par la matière organique dont les concentrations ont chuté au cours du compostage pour se maintenir à $27 \%$ pour T0, entre 19,3 et $23,1 \%$ pour les composts ayant comme co-substrat les déchets maraîchers et, entre 27,1 et $32,1 \%$ pour les composts ayant comme co-substrat les déchets de poissons. Le rapport AH/AF est inférieur à la valeur limite (> 2,5). Les concentrations en AF de tous les composts sont tous conformes à la valeur limite $(\leq 12,5$ $\mathrm{g} / \mathrm{kg}$ ) confirmant ainsi la bonne stabilité de la matière organique dans les différents composts.

\section{Qualité sanitaire des composts Concentrations en coliformes fécaux}

Les taux de réduction des coliformes fécaux dans les différents andains sont consignés dans le Tableau 2. Les taux de réduction enregistrés à partir du nombre de coliformes fécaux contenu dans les boues brutes non compostées, varient entre 11 et $73 \%$ dans les différents andains.

\section{Concentrations en œufs d'Ascaris}

Les concentrations en œufs d'Ascaris dans les différents andains sont mentionnées dans le Tableau 3. Les composts T0, T1a, T2a, T3a et T1b ont des concentrations de 3,4 à 1,2 supérieures à la recommandation de l'OMS de moins d'un œuf d'helminthes par gramme de compost. Dans les andains T2b et T3b, les concentrations en œufs d'Ascaris sont inférieures à la recommandation de l'OMS. 


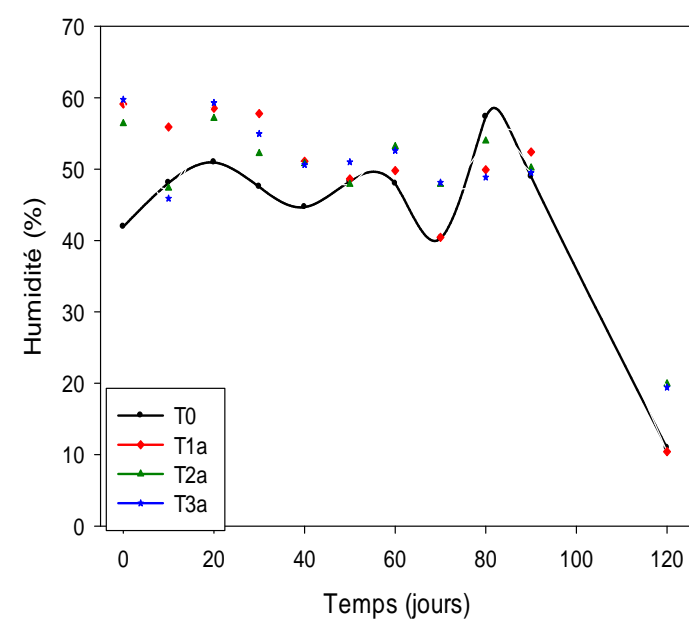

(a)

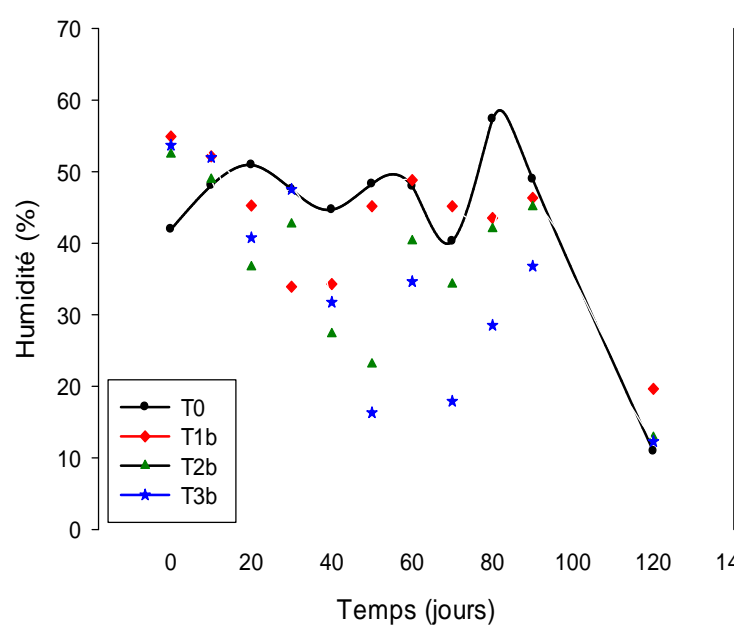

(b)

T0 : Témoin, uniquement boues de vidange (BV), T1a : mélange de 2 volumes BV + 1 volume de déchets maraîchers (DM), T2a : mélange de 1 volume $\mathrm{BV}+1$ volume $\mathrm{DM}, \mathrm{T} 3 \mathrm{a}$ : mélange de 1 volume $\mathrm{BV}+2$ volumes $\mathrm{DM}, \mathrm{T} 1 \mathrm{~b}$ : mélange de 2 volumes $\mathrm{BV}+1$ volume de déchets poissons (DP), T2b : mélange de 1 volume $\mathrm{BV}+1$ volume DP, T3b : mélange de 1 volume $\mathrm{BV}+2$ volumes DP.

Figure 1 : Teneur en eau au cours du processus de co-compostage a) de boues de vidange et de déchets maraichers et (b) de boues de vidange et de déchets de poissons.

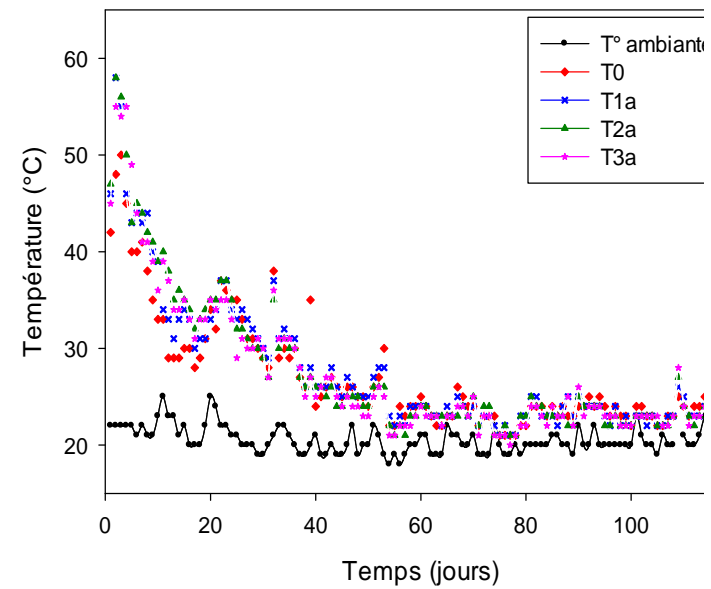

(a)

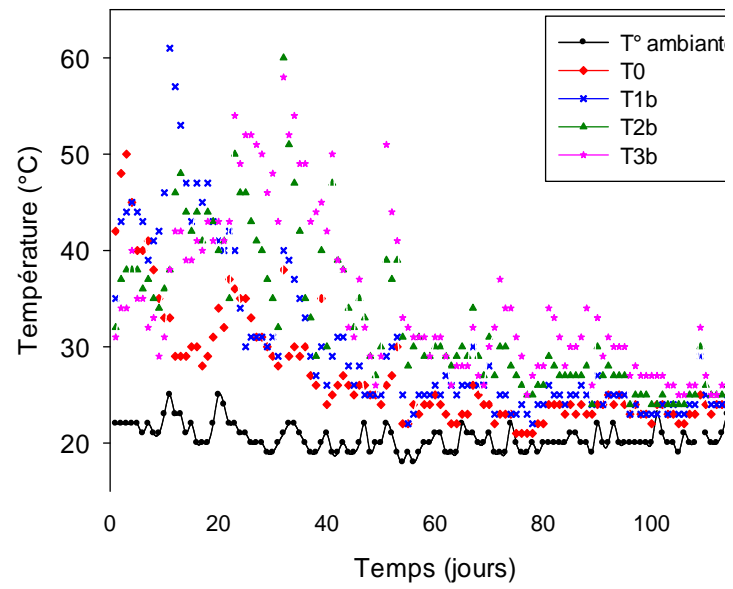

(b)

: Période retournement

T0 : Témoin, uniquement boues de vidange (BV), T1a : mélange de 2 volumes BV + 1 volume de déchets maraîchers (DM), T2a : mélange de 1 volume $\mathrm{BV}+1$ volume DM, T3a : mélange de 1 volume $\mathrm{BV}+2$ volumes DM, T1b : mélange de 2 volumes $\mathrm{BV}+1$ volume de déchets poissons (DP), T2b : mélange de 1 volume $\mathrm{BV}+1$ volume $\mathrm{DP}, \mathrm{T} 3 \mathrm{~b}$ : mélange de 1 volume $\mathrm{BV}+2$ volumes DP.

Figure 2: Evolution de la température lors du co-compostage (a) de boues de vidange et de déchets maraichers et (b) de boues de vidange et de déchets de poissons. 


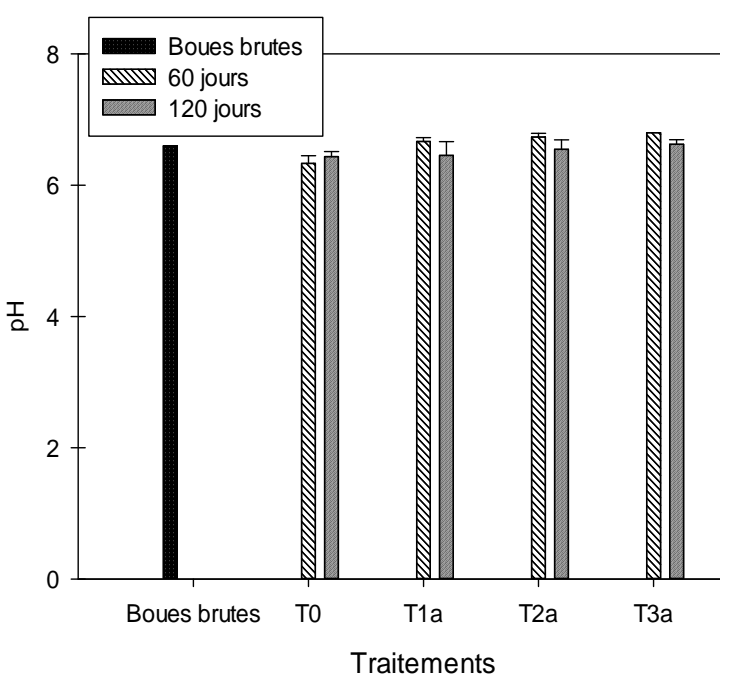

(a)

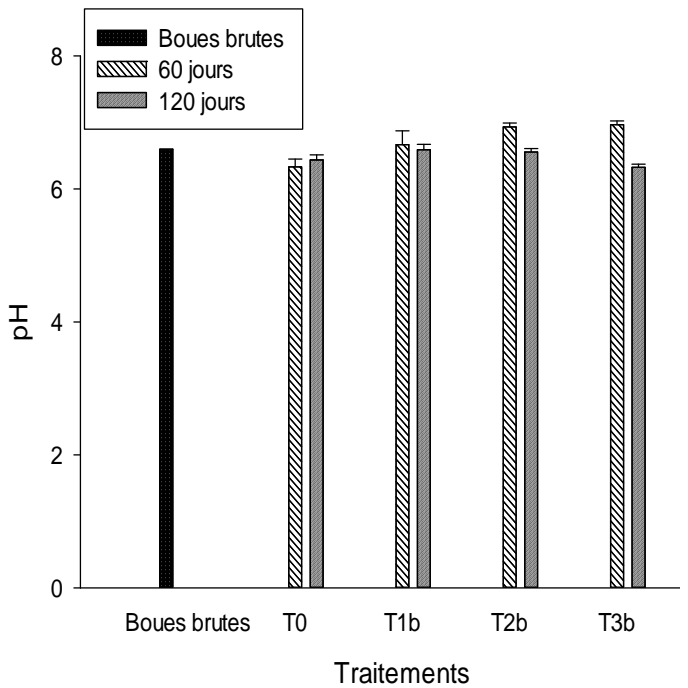

(b)

T0 : Témoin, uniquement boues de vidange (BV), T1a : mélange de 2 volumes BV + 1 volume de déchets maraîchers (DM), T2a : mélange de 1 volume $\mathrm{BV}+1$ volume DM, T3a : mélange de 1 volume $\mathrm{BV}+2$ volumes $\mathrm{DM}, \mathrm{T} 1 \mathrm{~b}$ : mélange de 2 volumes $\mathrm{BV}+1$ volume de déchets poissons (DP), T2b : mélange de 1 volume $\mathrm{BV}+1$ volume $\mathrm{DP}, \mathrm{T} 3 \mathrm{~b}$ : mélange de 1 volume $\mathrm{BV}+2$ volumes DP.

Figure 3: Evolution du pH à 2 mois et à 4 mois de co-compostage (a) de boues de vidange et de déchets maraichers (b) de boues de vidange et de déchets de poissons.

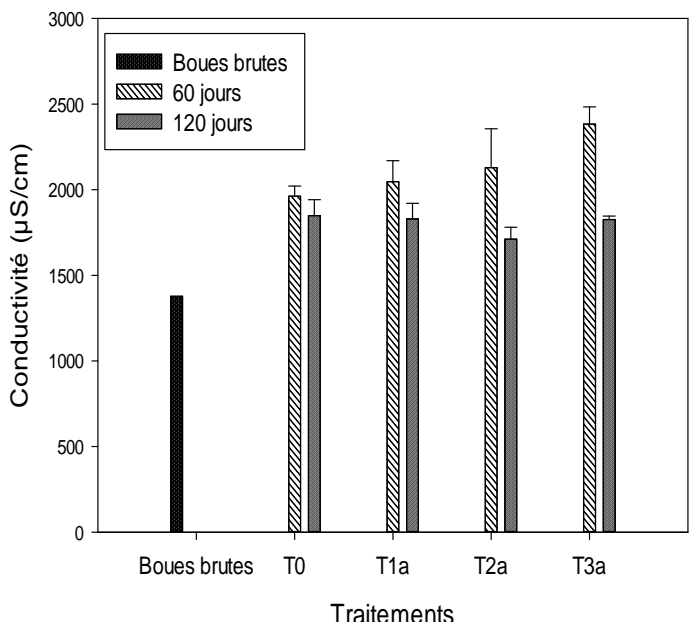

(a)

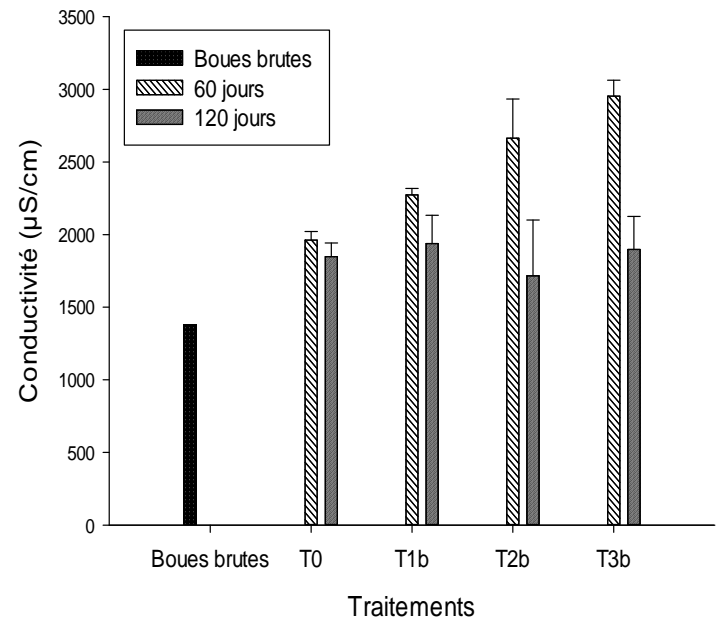

(b)

T0 : Témoin, uniquement boues de vidange $(\mathrm{BV}), \mathrm{T} 1 \mathrm{a}$ : mélange de 2 volumes $\mathrm{BV}+1$ volume de déchets maraîchers (DM), T2a : mélange de 1 volume $\mathrm{BV}+1$ volume DM, T3a : mélange de 1 volume $\mathrm{BV}+2$ volumes $\mathrm{DM}, \mathrm{T} 1 \mathrm{~b}$ : mélange de 2 volumes $\mathrm{BV}+1$ volume de déchets poissons (DP), T2b : mélange de 1 volume $\mathrm{BV}+1$ volume DP, T3b : mélange de 1 volume $\mathrm{BV}+2$ volumes DP.

Figure 4 : Variation de la conductivité au cours du co-compostage (a) de boues de vidange et de déchets maraichers et (b) de boues de vidange et de déchets de poissons. 


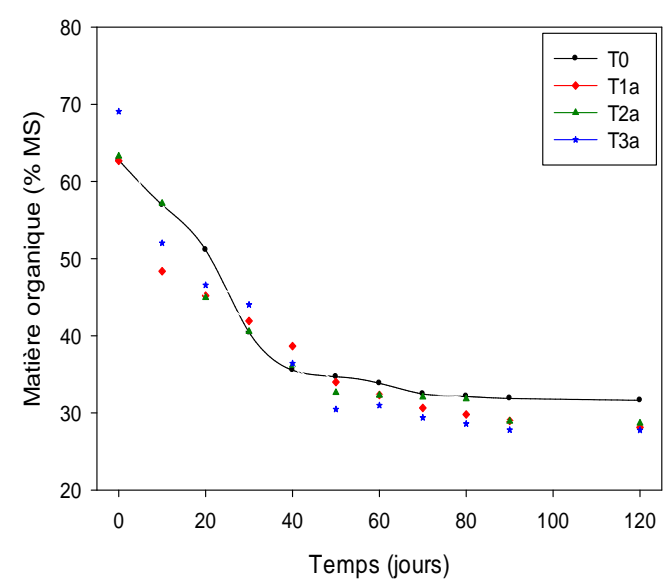

(a)

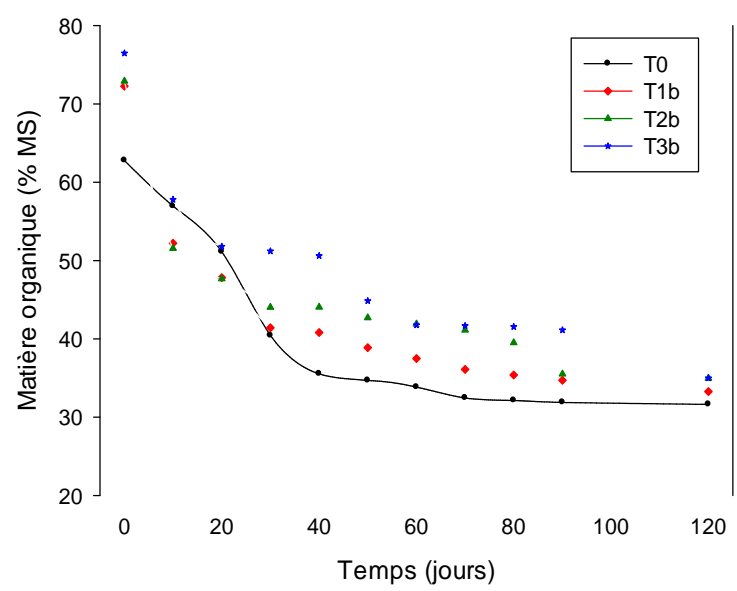

(b)

T0 : Témoin, uniquement boues de vidange (BV), T1a : mélange de 2 volumes BV + 1 volume de déchets maraîchers (DM), T2a : mélange de 1 volume BV +1 volume DM, T3a : mélange de 1 volume BV +2 volumes DM, T1b : mélange de 2 volumes $\mathrm{BV}+1$ volume de déchets poissons (DP), T2b : mélange de 1 volume $\mathrm{BV}+1$ volume $\mathrm{DP}, \mathrm{T} 3 \mathrm{~b}$ : mélange de 1 volume $\mathrm{BV}+2$ volumes DP.

Figure 5 : Evolution de la matière organique au cours du co-compostage (a) de boues de vidange et de déchets maraichers et (b) de boues de vidange et de déchets de poissons.

Tableau 1: Qualité agronomique des composts des différents andains.

\begin{tabular}{|c|c|c|c|c|c|c|c|c|}
\hline Paramètres & Unités & T0 & T1a & T2a & T3a & T1b & $\mathbf{T} 2 \mathbf{b}$ & T3b \\
\hline \multicolumn{9}{|c|}{ Physico-chimique } \\
\hline $\mathrm{pH}(1: 2,5)$ & & 6,4 & 6,5 & 6,5 & 6,6 & 6,6 & 6,6 & 6,3 \\
\hline EC $(1: 10)$ & $\mu \mathrm{s} / \mathrm{cm}$ & 1849 & 1829 & 1712 & 1826 & 1938 & 1716 & 1898 \\
\hline \multicolumn{9}{|l|}{ Stabilité } \\
\hline $\mathrm{C}$ & $\%$ & 15,6 & 13,4 & 12,9 & 11,2 & 15,2 & 18 & 18,6 \\
\hline MO & $\%$ & 27 & 23,1 & 22,2 & 19,3 & 26,1 & 31,0 & 32,1 \\
\hline $\mathrm{C} / \mathrm{N}$ & & 10,6 & 9,5 & 9,2 & 8,6 & 9,0 & 9,5 & 8,4 \\
\hline $\mathrm{AH}$ & $\%$ & 0,9 & 1 & 1 & 1,3 & 1,7 & 2 & 3,6 \\
\hline $\mathrm{AF}$ & $\%$ & 8,35 & 12,5 & 12,1 & 11,4 & 9,8 & 9,4 & 11,3 \\
\hline $\mathrm{AH} / \mathrm{AF}$ & & 0,11 & 0,1 & 0,1 & 0,11 & 0,18 & 0,21 & 0,32 \\
\hline \multicolumn{9}{|l|}{ Nutriments } \\
\hline \multirow[t]{2}{*}{$\mathrm{N}$} & $\%$ & 1,5 & 1,4 & 1,4 & 1,3 & 1,7 & 1,9 & 2,2 \\
\hline & $\mathrm{Kg} / \mathrm{T}$ & 15 & 14 & 14 & 13 & 17 & 19 & 22 \\
\hline \multirow[t]{2}{*}{$\mathrm{P}$} & $\%$ & 0,3 & 0,5 & 0,3 & 0,2 & 0,5 & 0,4 & 0,5 \\
\hline & $\mathrm{Kg} / \mathrm{T}$ & 3,3 & 5 & 3,3 & 2,4 & 5 & 4,3 & 5 \\
\hline \multirow[t]{2}{*}{$\mathrm{K}$} & $\%$ & 0,07 & 0,12 & 0,2 & 0,3 & 0,2 & 0,3 & 0,3 \\
\hline & $\mathrm{Kg} / \mathrm{T}$ & 0,7 & 1,2 & 2 & 3 & 2 & 3 & 3 \\
\hline $\mathrm{CaO}$ & $\%$ & 4,78 & 3,9 & 3,4 & 2,76 & 3,3 & 2,32 & 1,7 \\
\hline $\mathrm{MgO}$ & $\%$ & 0,47 & 0,52 & 0,54 & 0,57 & 0,69 & 0,79 & 0,84 \\
\hline $\mathrm{Na} 2 \mathrm{O}$ & $\%$ & 0,02 & 0,03 & 0,03 & 0,03 & 0,04 & 0,05 & 0,05 \\
\hline
\end{tabular}


Tableau 2 : Concentration et taux de réduction des coliformes fécaux dans les différents andains.

\begin{tabular}{llllllllll}
\hline & Unité & $\begin{array}{l}\text { Boue } \\
\text { brute }\end{array}$ & T0 & T1a & T2a & T3a & T1b & T2b & T3b \\
\hline $\begin{array}{l}\text { Concentrations en } \\
\text { coliformes fécaux }\end{array}$ & UFC/100g & 37000 & 29000 & 20000 & 16000 & 10000 & 10000 & 22000 & 33000 \\
$\begin{array}{l}\text { Taux de réduction } \\
\text { Taun }\end{array}$ & & 22 & 46 & 57 & 73 & 73 & 40 & 11 \\
\hline
\end{tabular}

T0 : Témoin, uniquement boues de vidange (BV), T1a : mélange de 2 volumes BV + 1 volume de déchets maraîchers (DM), T2a : mélange de 1 volume BV +1 volume DM, T3a : mélange de 1 volume $\mathrm{BV}+2$ volumes DM, T1b : mélange de 2 volumes $\mathrm{BV}+1$ volume de déchets poissons $(\mathrm{DP}), \mathrm{T} 2 \mathrm{~b}$ : mélange de 1 volume $\mathrm{BV}+1$ volume $\mathrm{DP}, \mathrm{T} 3 \mathrm{~b}$ : mélange de 1 volume $\mathrm{BV}+2$ volumes DP.

Tableau 3 : Concentrations en œufs d'Ascaris dans les andains.

\begin{tabular}{|c|c|c|c|}
\hline \multirow[t]{2}{*}{ Echantillons } & \multicolumn{3}{|c|}{ Nombre d'œufs d'Ascaris/g de compost } \\
\hline & Vivant & Inactif & Total \\
\hline T0 & 3,4 & 12,7 & 16,1 \\
\hline T1a & 2,5 & 10,1 & 12,2 \\
\hline $\mathrm{T} 2 \mathrm{a}$ & 2,8 & 8,2 & 11 \\
\hline $\mathrm{T} 3 \mathrm{a}$ & 2,2 & 7,5 & 9,7 \\
\hline $\mathrm{T} 1 \mathrm{~b}$ & 1,2 & 2,8 & 4 \\
\hline $\mathrm{T} 2 \mathrm{~b}$ & 0,7 & 2,9 & 3,6 \\
\hline $\mathrm{T} 3 \mathrm{~b}$ & 0,3 & 3 & 3,3 \\
\hline
\end{tabular}

\section{DISCUSSION}

Suivi du processus de compostage E volution de l'humidité

Les niveaux d'humidité des traitements des boues de vidange avec les déchets maraichers sont comparables à ceux trouvés par plusieurs auteurs dans des andains de cocompostage de boues d'épuration et de déchets organiques (Cofie et al., 2009; Fourti, 2013; Temgoua et al., 2014). Ils ont trouvé dans leurs différentes études que l'humidité dans les andains variait entre 30 et $70 \%$. Parmi ces auteurs, d'autres comme Fourti, (2013) ont noté que l'humidité dans les andains devrait être autour de 40 à $60 \%$ pour un compostage optimal. Cet auteur a, par ailleurs, affirmé qu'un pourcentage d'humidité élevé favorisait une décomposition anaérobie, tandis qu'une faible teneur en humidité ralentissait le processus de compostage et que les micro-organismes mouraient ou entraient en dormance. Dans les andains de boues de vidange et des déchets de poisson, l'humidité est faiblement intégrée dans les mco-substrats. Cette variation de l'humidité peut être liée aux caractéristiques du matériau. En effet, dans les échantillons prélevés au niveau des andains ayant comme co-substrat les déchets de poissons, il y'a beaucoup de déchets solides persistants comme les os et les écailles de poissons. Ces derniers peuvent contribuer à augmenter le taux de Matières Sèches d'où une humidité faible. Cependant les andains co-compostés 
avec les déchets de poissons en fin de compostage ont des taux d'humidité beaucoup plus élevés que ceux co-compostés avec les déchets maraîchers. Les taux d'humidité élevés dans les andains contenant des déchets de poissons, peuvent être liés à leurs forts taux de matières grasses (Gea et al., 2007; Ruggieri et al., 2008a; Ruggieri et al., 2008b) qui sont des produits insolubles dans l'eau.

\section{Evolution de la température}

Dans l'ensemble, l'évolution des températures est similaire pour les différents échantillons d'andains (T1a, T2a, T3a) et même pour le témoin (T0). La différence des andains co-compostés ne semble pas avoir une influence sur les processus de dégradation de la matière organique lesquels sont responsables des variations de la température. Ces résultats sont similaires à ceux de Cofie et al. (2009). En effet, ces auteurs travaillant sur des doses de $2 / 1$ et $3 / 1$ en volume de déchets solides organiques et de boues de vidange domestiques ont montré que l'évolution de la température était similaire dans les différents andains. Toutefois, les températures enregistrées dans cette étude au cours de la phase thermophile sont inférieures à celles trouvées dans la littérature (Albrecht, 2007; Cofie et al., 2009; Temgoua et al., 2014, Biekre, 2018). Ces auteurs ont trouvé dans leurs différentes études que les températures au cours de la phase thermophile étaient supérieures à $60{ }^{\circ} \mathrm{C}$. Les faibles températures enregistrées dans notre étude peuvent être liées aux faibles volumes des andains. En effet, selon Temgoua et al. (2014) les andains doivent avoir des tailles de 5 à 6 tonnes d'ordures pour que la température puisse atteindre 65 à $70{ }^{\circ} \mathrm{C}$ au bout d'une semaine de compostage. Dans notre étude les andains avaient des tailles de $0,15 \mathrm{~m}^{3}$. Les déchets de poissons, ralentissent donc les processus de dégradation de la matière organique. Ce ralentissement pourrait provenir de la forte teneur en protéine et en matières grasses. En effet selon Mc Clintock (2005), ces deux composés organiques ont des vitesses de dégradation beaucoup plus lentes que celles des glucides et des sucres. Ces résultats sont en adéquation avec les travaux de Gea et al.
(2007) qui ont soutenu que la haute teneur en énergie chimique contenue dans les matières grasses est responsable de la longue phase thermophile. Toutefois, les températures maximales enregistrées sont proches quelle que soit la composition du mélange. Les hautes températures enregistrées durant cette période sont dues à la forte activité lipolytique (Gea et al., 2007) des matières grasses contenues dans les déchets de poissons. Des études antérieures ont, montré qu'une température de 50 à $60{ }^{\circ} \mathrm{C}$ pendant la phase thermophile est un bon indicateur d'un compostage efficace (Capizzi-Banas et al., 2004). Après la phase thermophile, la température dans les andains a progressivement baissé jusqu'au niveau de la température ambiante comme dans les travaux de Baba Ardi., 2018. Toutefois l'atteinte de cette température ambiante est plus lente dans les andains co-compostés avec les déchets de poissons. Par ailleurs, l'évolution de la température en fonction du temps de compostage a montré que celle-ci enregistrait des hausses plus ou moins importantes après chaque retournement comme dans les travaux de certains auteurs (Temgoua, 2014 ; Bacar., 2015 ; Baba Ardi, 2018). Ces constats sont faits aussi au niveau de tous les andains. Ceci peut être lié au fait que selon Cofie et al. (2009), les retournements périodiques permettent de remettre la matière organique et aussi d'apporter l'oxygène à l'intérieur de l'andain. Lors des retournements, l'humidité est aussi ajustée à des niveaux permettant le maintien de l'activité des microorganismes.

\section{Variations $d u$ pH}

Dans notre étude, les valeurs de $\mathrm{pH}$ trouvées sont dans les mêmes proportions que celles obtenues par ces auteurs montrant ainsi une maturité de ce compost. En réalité l'évolution du $\mathrm{pH}$ dépend considérablement des substrats et additifs éventuels composant le mélange initial (Eklin et Kirchmen, 2000). La baisse du pH peut s'expliquer par la production d'acides organiques suite à la dégradation des glucides, des lipides et d'autres substances (Znaïdi, 2002). Aussi la production de $\mathrm{CO}_{2}$ lors de la dégradation aérobie contribue à l'acidification du milieu 
par sa dissolution dans l'eau et la production d'acide carbonique (Znaïdi, 2002).

Par ailleurs, cette baisse est beaucoup plus prononcée au niveau des andains ayant comme co-substrat les déchets de poissons (T1b, T2b, T3b). La diminution sensible de la valeur du $\mathrm{pH}$ enregistré dans ces andains peut être due à la formation d'acides gras au cours de la décomposition de la matière organique selon Ammari et al. (2012) qui ont obtenu des résultats similaires. Le $\mathrm{pH}$ n'est donc pas un critère facile à utiliser pour suivre le déroulement du compostage (Yulipriyanto, 2001)

\section{Variation de la conductivité}

Le compostage a donc entrainé une augmentation de la conductivité au début du processus. Ceci peut être lié au fait que le compostage en favorisant la dégradation de la matière organique favorise la libération de matières minérales qui contribuent à augmenter la conductivité électrique. Selon Ammari et al. (2012), l'augmentation de la conductivité électrique (CE) est induite par l'addition de quantités importantes de l'agent de charge; c'est-à-dire, dans le cas de mélange de haut rapport $\mathrm{C} / \mathrm{N}$, à la matière de boues. Une diminution de la conductivité entre 2 mois et 4 mois de compostage dans tous les différents andains est notable au cours du compostage. Les faibles teneurs en sels dans les boues de vidange brutes issues des fosses septiques pourraient être à l'origine de cette baisse.

\section{Evolution de la matière organique}

La principale raison de cette diminution est l'utilisation par les microorganismes des substances organiques indispensables à leur métabolisme (Francou, 2003). Parmi ces substances, le carbone est considéré comme source de nourriture et l'azote comme une enzyme digestive (Fourti, 2013). La diminution de la teneur en matières organiques est beaucoup plus rapide dans les andains co-compostés avec les déchets maraîchers que dans ceux co-compostés avec les déchets de poissons. Les déchets de poissons sont plus riches en matières grasses et en protéines que les déchets maraîchers d'origine végétale. Ainsi, selon McClintock
(2005), la baisse assez lente de la quantité de matière organique dans les andains $\mathrm{T} 1 \mathrm{~b}, \mathrm{~T} 2 \mathrm{~b}$ et $\mathrm{T} 3 \mathrm{~b}$ peut être liée à la vitesse de dégradation plus lente des composés gras et protéiques par rapport aux sucres et glucides.

\section{Qualité agronomique des composts}

La plupart des paramètres suivis sont en accord avec les travaux réalisés dans le cocompostage des boues de vidange avec des déchets organiques d'origine végétale et animale (Cofie et al., 2009; López-Mosquera et al., 2011). Tous les composts ont des $\mathrm{pH}$ légèrement acides à neutre se trouvant dans la gamme définit par Ammari et al. (2012) pour les composts matures. Tous les biosolides ont des rapports $\mathrm{C} / \mathrm{N}$ inférieurs à 12 comme dans les travaux d'El Fels (2014) et de Biekre (2018). Ces composts peuvent donc être caractérisés de stable car, selon Bernal et al.(2009) le rapport $\mathrm{C} / \mathrm{N}$ de compost stable doit être inférieur à 12. Les quantités de matières organiques sont en baisse au cours du compostage et ont atteint des valeurs similaires trouvées dans des composts stables de boues de vidange et de déchets verts (Cofie et al., 2009). Toutefois, pour les déchets de poissons, les concentrations en matière organique dans le produit final sont largement inférieures à celles trouvées dans la littérature par Ruggieri et al. (2008a) sur des composts de boues d'épuration et de matières grasses animales et par López-Mosquera et al. (2011) sur des composts de déchets de poissons et de fruits de mer. Toutefois les concentrations en $\mathrm{AF}$ de tous les composts sont tous conformes à la valeur limite définie par Bernal et al. (2009) confirmant ainsi la bonne stabilité de la matière organique dans les différents composts. Les composts ont une valeur agronomique élevée. Leurs concentrations en divers éléments tels que l'azote, le phosphore, le potassium, le Calcium et le Magnésium sont comparables et assez élevées pour tous les composts. Ces concentrations sont comparables à celles trouvées par beaucoup d'auteurs dans la littérature (Ruggieri et al., 2008a; Cofie et al., 2009; López-Mosquera et al., 2011). Toutefois, les quantités de Sodium sont faibles pour tous les composts. Ceci 
montre que les composts ont des salinités faibles; ce qui est un bon résultat pour leur utilisation dans n'importe quel type de sol. L'utilisation d'un tel compost peut être bénéfique à court terme par l'apport assez important d'éléments nutritifs nécessaires au développement des plantes.

\section{Qualité sanitaire des composts}

La qualité sanitaire est déterminée à travers les concentrations en coliformes fécaux et en œufs d'Ascaris.

\section{Concentrations en coliformes fécaux}

Toutefois, les taux de réduction enregistrés sont largement supérieurs à ceux trouvés par Mainoo (2007), Ndegwa et Thompson (2001) et El Fels (2014) qui ont estimé que le compostage permet une élimination totale des germes pathogènes. En effet, dans un processus de compostage aérobie où les températures avoisinent les 60 ${ }^{\circ} \mathrm{C}$ pendant plusieurs semaines, il se produit une destruction efficace des agents pathogènes selon Compaoré et al. (2010). Dans notre étude des températures du même ordre entre 50 et $63{ }^{\circ} \mathrm{C}$ obtenues dans les différents andains auraient dû entrainer une forte réduction des pathogènes. La présence de ces germes a certainement été favorisée par la présence d'animaux et d'insectes (dans la zone d'étude) qui se réfugient dans les andains comme l'ont montré Compaoré et al. (2010).

\section{Concentrations en œufs d'Ascaris}

Le compostage n'a pas permis d'éliminer le risque parasitaire. Pourtant cette technique est connue pour son action positive dans l'inactivation des œufs de parasites (Bacar, 2015). En effet, Maya et al. (2012) ont montré qu'une élévation de température à des niveaux supérieurs à $45^{\circ} \mathrm{C}$, permet d'inactiver toutes les espèces de parasites au bout de 6 jours. Toutefois l'auteur a précisé que l'action de la température n'est efficace que dans des conditions de $\mathrm{pH}$ de 5,3 et de siccité de $90 \%$. Dans notre étude, les températures obtenues sont supérieures à $40{ }^{\circ} \mathrm{C}$ pendant plus de 6 jours; ce qui aurait dû entrainer une inactivation des œufs de parasites. Malheureusement tel n'a pas été le cas avec des concentrations en œufs d'Ascaris se situant entre 1,2 et 3,4 œufs/g de compost dans les andains T0, T1a, T2a, T3a et T1b. Les concentrations encore élevées en parasites dans ces andains peuvent être dues aux $\mathrm{pH}$ qui sont tous supérieurs à 5,3 ou aux siccités faibles inférieures à 90\%. Dans les andains $\mathrm{T} 2 \mathrm{~b}$ et $\mathrm{T} 3 \mathrm{~b}$, les concentrations en œufs d'Ascaris sont conformes aux recommandations de l'OMS. Pourtant dans ces andains, les conditions de $\mathrm{pH}$ et de siccité sont comparables à ceux des autres andains dont les concentrations sont supérieures à la recommandation OMS. La bonne inactivation des œufs dans ces andains peut être due à des facteurs de stress biologique. En effet, selon Sene (2008), les acides organiques, les aldéhydes et les alcools se sont révélés agir comme des désinfectants des pathogènes dans les biosolides. Ces facteurs de stress biologique peuvent être à l'origine de l'inactivation des œufs d'helminthes dans ces andains constitués pour moitié ou pour majorité de déchets de poissons. Dans l'andain T1b, les concentrations encore élevées en œufs d'Ascaris peuvent être dues au fait que dans ce tas, la quantité de déchets de poisson étant faible, les quantités d'acides organiques, d'aldéhydes et d'alcools sont aussi faibles ce qui n'a pas permis d'inactiver les œufs de parasites jusqu'à des niveaux compatibles avec les recommandations de l'OMS.

\section{Conclusion}

A Dakar comme beaucoup de grandes villes en Afrique de l'ouest, la gestion des boues de vidange combinée à la croissance démographique constitue un problème d'une grande envergure surtout avec la nouvelle pratique de certains maraichers avec l'utilisation directe de ces effluents gorgés d'agents pathogènes. Cette étude sur la valorisation des boues de vidange par la technique de co-compostage contribue à l'amélioration du cadre de vie des populations tout en sécurisant l'utilisation de ces produits. Les résultats de cette étude ont montré que tous les composts sont matures. Les paramètres de maturité tels que la température, la matière organique, et 
l'humidité sont conformes à ceux d'un compost mature. Aussi les concentrations en éléments nutritifs sont assez élevées. Toutefois, ces composts ne sont pas sains du point de vue sanitaire avec des concentrations en coliformes fécaux supérieures aux normes internationales. De plus, les concentrations en œufs d'ascaris sont supérieures à la recommandation de l'OMS dans le compost témoin T0. Toutefois, dans les composts constitués pour moitié (T2b) ou pour majorité de déchets de poisson (T3b), les œufs d'ascaris sont inactivés jusqu'à des niveaux compatibles avec les recommandations OMS. De ce fait, l'ajout de déchets riches en graisses et/ou en protéines aux déchets à faible teneur en énergie peut être conseillé pour répondre aux exigences internationales en termes de qualité sanitaire des composts. Toutefois, la gestion de l'humidité devra être le point crucial dans la gestion de tels composts. Le stockage pour un délai supplémentaire à l'abri d'agents contaminants externes peut être recommandé avant utilisation pour les autres composts.

\section{CONFLIT D'INTERETS}

Les auteurs déclarent n'avoir aucun conflit d'intérêts pour cet article.

\section{CONTRIBUTIONS DES AUTEURS}

Tous les auteurs ont contribué à la réalisation de ce travail et à la rédaction du manuscrit.

\section{REMERCIEMENTS}

Les auteurs remercient le laboratoire de l'Institut National de Pédologie (INP) du Sénégal et le laboratoire de l'Office National de l'Assainissement du Sénégal (ONAS) pour leurs contributions dans la réalisation des analyses des différents paramètres ayant permis de caractériser les composts. Les auteurs remercient par ailleurs l'ONAS pour avoir hébergé l'équipe des chercheurs.

La conception et la planification de l'étude ont été rendues possibles grâce à l'appui de l'équipe de chercheurs du Laboratoire d'Etude Environnementales des Milieux Urbains (LEEMUR) de l'Institut des
Sciences de l'Environnement (ISE) de l'Université Cheikh Anta Diop de Dakar (UCAD).

\section{RÉFÉRENCES}

ADEME. 2006. Les bases du co-compostage à la ferme: Fiche B: Procédé de compostage, co-compostage, cocompostage à la ferme.

Albrecht R. 2007. Co-compostage de boues de stations d'épuration et de déchets verts: Nouvelle méthodologies du suivi des transformations de la matière organique. Thèse de doctorat, Université PAUL CEZANNE AIX-MARSEILLE III: p. 189.

Ammari TG, Al-Omari Q, Abbassi B. 2012. Composting sewage sludge amended with different sawdust proportions and textures and organic waste of food industry - assessment of quality. Environmental Technology, 33: 16411649.

DOI:

10.1080/09593330.2011.641589.

ANSD. 2013. Enquête démographique et de santé continue au Sénégal (EDSContinue) 2012-2013. Agence Nationale de la Statistique et de la Démographie (ANSD), Ministère de l'Economie et des Finances, République du Sénégal. 215p.

APHA. 2005. Standards Methods for Examination of Water and Wastewater (21st edn). American Public Health Association, American Water Work Association, Water Environmental Federation. Washington DC.

Baba Ardi H. 2018. Etude de l'intérêt environnemental et agronomique de compost de déchets oasiens. Mémoire de Master, Université Kasdi Merbah Ouargla, Algérie.

Bacar A. 2015. Valorisation des boues de vidange dans l'agriculture: Etude du cocompostage de boues de vidange de Cambérène (Dakar/Sénégal) et de feuilles de Typha domongensis Pers. Mémoire de Master, Université Cheikh Anta Diop Dakar, Dakar.

Bernal MP, Alburquerque JA, Moral R. 2009. Composting of animal manures and 
chemical criteria for compost maturity assessment. A review. Bioresource Technology, 100: 5444-5453. DOI: 10.1016/j.biortech.2008.11.027.

Biekre AHT, Tie BT, Dogbo DO. 2018. Caaractéristiques physico-chimiques des composts à base de sous produits de ferme de Songon en Côte d'Ivoire. International Journal of Biological and Chemical Sciences, 12(1): 596-609. DOI: http://dx.doi.org/10.4314/ijbcs.v12i1.45.

Capizzi-Banas S, Deloge M, Remy M, Schwartzbrod J. 2004. Liming as an advanced treatment for sludge sanitisation: helminth eggs eliminationAscaris eggs as model. Water Research, 38: $3251-3258 . \quad$ DOI : 10.1016/j.watres.2004.04.015.

Cofie O, Kone D, Rothenberger S, Moser D, Zubruegg C. 2009. Co-composting of faecal sludge and organic solid waste for agriculture: Process dynamics. Water Resaerch, 43: 4665 - 4675. DOI: 10.1016/j.watres.2009.07.021.

Compaoré E, Nanema LS, Bonkoungou S, Sedogo MP. 2010. Évaluation de la qualité de composts de déchets urbains solides de la ville de Bobo-Dioulasso, Burkina Faso pour une utilisation efficiente en agriculture. Applied Biosciences, 33: 2076 - 2083.

Dème N. 2008. Traitement de boues de vidange de système d'assainissement autonome à Dakar: évaluation de l'efficacité de la séparation solide/liquide dans deux bassins expérimentaux de sédimentation/épaississement. Mémoire de DEA, Université Cheikh Anta Diop de Dakar, Dakar. p. 102.

Diawara AB. 2009. Les déchets solides à Dakar. Environnement, sociétes et gestion urbaine. Thèse de doctorat, Université Bordeaux III de Montaigne. p. 792.

Dème N. 2008. Traitement de boues de vidange de système d'assainissement autonome à Dakar: évaluation de l'efficacité de la séparation solide/liquide dans deux bassins expérimentaux de sédimentation/épaississement. Mémoire de DEA, Université Cheikh Anta Diop de Dakar, Dakar, p. 102.

Eklin Y, Kirchmann H. 2000. Composting and storage of organic household waste with different litter amendments I: carbon turnover. Bioresource Technology, 74(2): 115-124. DOI: http://doi.org/10.1016/S09608524(00)00004-3.

El Fels L. 2014: Suivi physico-chimique, microbiologique et ecotoxicologique du compostage de boues STEP mélangées à des déchets de palmier. Thèse de doctorat, Université Cadi Ayyad, Marrakech.

Fourti O. 2013. The maturity tests during the composting of municipal solid wastes. Review. Resources, Conservation and Recycling, 72: 43-49. DOI : 10.1016/j.resconrec.2012.12.001.

Francou C. 2003. Stabilition de la matière organique au cours du compostage de déchets urbains: Influence de la nature des déchets et du procédé de compostage - Recherche d'indicateurs pertinents. Thèse de Doctorat. Institut National Agronomique, Ecole doctorale Abies. $244 \mathrm{p}$.

Gaye M, Niang S. 2010. Manuel des bonnes pratiques de l'utilisation saine des eaux usées dans l'agriculture urbaine. Dakar.

Gea T, Ferrer P, Alvaro G, Valero F, Artola A, Sànchez A. 2007. Co-composting of sewage sludge:fats mixtures and characteristics of the lipases involved. Biochemical Engineering Journal, 33 275-283.

DOI: 10.1016/j.bej.2006.11.007.

Gning JB, Diop C, Dongo K, Koné D. 2017. Facteurs déterminants le tarif de la vidange mécanique des matières de boues d'assainissement à Dakar. International Journal Biological and Chemical Sciences, 11(1): 313-332. DOI: http://dx.doi.org/10.4314/ijbcs.v11i1.25

Keffala C, Harerimana C, Vasel JL. 2012. Oeufs d'helminthes dans les eaux usées 
et les boues de station d'épuration: enjeux sanitaires et intérêt du traitement par lagunage. Environnement, Risques \& Sante, 11(6): 511-520. DOI : 10.1684/ers.2012.0573.

Kone M, Service E, Ouattara Y, Ouattara P, Bonou L, Joly P. 2016. Caractérisation des boues de vidange dépotées sur les lits de séchage de Zagtouli (Ouagadougou). International Journal of Biological and Chemical Sciences, 10(6): 2781-2795. DOI: $10.4314 /$ ijbcs.v10i6.30.

Kouawa T. 2017. Traitement des boues de vidange par lits de séchage sous climat soudano-sahélien. Thèse de doctorat. Université de Strasbourg, Strasbourg.

López-Mosquera ME, Fernández-Lemaa E, Villaresa R, Corralb R, Alonsob B, Blancob C. 2011. Composting fish waste and seaweed to produce a fertilizer for use in organic agriculture. Procedia Environmental Sciences, 9: 113 - 117. DOI : 10.1016/j.proenv.2011.11.018.

Mainoo NK. 2007. Feasibility of low cost vermicompost production in Accra, Ghana. Thèse de Doctorat. University of Montreal, Montreal. p. 103.

Maya C, Torner-Morales FJ, Lucario ES, Hernandez E, Jimènez B. 2012. Viability of six species of larval and non-larval helminth eggs for different conditions of temperature, $\mathrm{pH}$ and dryness. Water Resaerch, 46: 4770-4782. DOI : 10.1016/j.watres.2012.06.014.

McClintock NC. 2005. Production de compost et usage dans les systèmes agricoles durables: Notes prises sur le terrain à l'intention des agriculteurs. Center for Environmental Farming Systems. Raleigh, NC 27695-7609. N 3:9 pp.

Milin S. 2012. Comparaison de deux méthodes spectrophotométriques de dosage de l'acide phosphorique: Application à des sols et des végétaux. Le cahier des techniques de l'INRA (77) $\mathrm{n}^{\circ} 3$.

Ndegwa PM, Thompson SA. 2001. Integrating composting and vermicomposting in the treatment and bioconversion of biosolids.
Bioressources Technology, 76(2): 107112 DOI: $10.1016 / \mathrm{s} 0960-$ 8524(00)00104-8.

Ndiaye ML. 2009. Impacts sanitaires des eaux d'arrosage de l'agriculture urbaine de Dakar (Sénégal). Thèse de doctorat. Université de Genève, Genève, p. 101.

Niang Y, Niang S, Niassy S, Dieng Y, Gaye ML, Diarra K. 2012. Urban agriculture in Senegal: effect of wastewater on the agronomical performance and hygien quality of tomato and lettuce. International Journal oj Biological and Chemical Sciences, 6(4): 1519-1526. DOI http://dx.doi.org/10.4314/ijbcs.v6i4.11.

Palm CA, Gachengo CN, Delve RJ, Cadisch G, Giller KE. 2001. Organic inputs for soil fertility management in tropical agroecosystems: Application of an organic resource database. Agric. Ecosyst. Environ., 83: 27-42. DOI : https://doi.org/10.1016/S01678809(00)00267-X.

Ruggieri L, Artola A, Gea T, Sanchez A. 2008a. Biodegradation of animal fats in a co-composting process with wastewater sludge. International Biodeterioration \& Biodegradation, 62: 297-303. DOI : https://doi.org/10.1016/j.ibiod.2008.02.0 04.

Ruggieri L, Gea T, Artola A, Sanchez A, Group GCR. 2008b. Influence of different co-substrates biochemical composition on raw sludge cocomposting. Biodegradation, 19: 403415. DOI : 10.1007/s10532-007-9146-2.

Seck A. 2016. Optimisation du séchage des boues de vidange domestiques de Dakar (Sénégal) par des lits de séchage non plantés pour une valorisation énergétique des biosolides : caractérisation physicochimique, calorifique et biologique, Thèse de doctorat Unique, Université Cheikh Anta Diop de Dakar, Dakar.

Sene T. 2008. Contribution à l'étude de la valorisation des co-produits de la sole tropicale (Cynoglossus senegalensis) après hydrolyse enzymatique. Thèse de 
doctorat, Université Cheich Anta Diop de Dakar, Dakar, p. 118.

Sonko EM. 2008. Traitement de boues de vidange de systèmes d'assainissement autonome à Dakar: évaluation de la séparation solide/liquide de lits de séchage non plantés soumis à différentes charges de boues domestiques. Mémoire DEA, Université Cheich Anta Diop de Dakar, Dakar, p. 72.

Sonko EM. 2015. Traitement de boues d'assainissement domestique par lits de séchage plantés: Performances du système en fonction du type et de la variabilité des boues, de la fréquence d'alimentation et de la nature des macrophytes. Thèse de doctorat unique, Institut des Sciences de l'Environnement, Université Cheikh Anta Diop Dakar, Dakar.
Temgoua E, Ngnikam E, Dameni H, Kouedeu-Kameni GS. 2014 Valorisation des ordures ménagères par compostage dans la ville de Dschang, Cameroun. Tropicultura, 32 (1): 28-36.

Yulipriyanto H. 2001. Emission d'effluents gazeux lors du compostage de substrats organiques en relation avec l'activite microbiologique (nitrification/denitrification). Thèse de doctorat, Université Rennes 1, Rennes.

Znaïdi IEA. 2002. Etude et évaluation du compostage de différents types de matières organiques et des effets des jus de composts biologiques sur les maladies des plantes. Master of Science degree, CIHEAM Mediterranien Agronomic Institute of Bari, Tunisie. 